\title{
Atypical Presentation and Disappearance of a Barrow Type A CCF With Glaucoma, Ptosis and 6th Nerve Palsy
}

\author{
Goktug Demirci ${ }^{\mathrm{a}, \mathrm{d}}$, Gokhan Gulkilik ${ }^{\mathrm{a}}$, Mustafa Eliacik ${ }^{\mathrm{a}}$, \\ Erol Demirci ${ }^{\mathrm{b}}$, Mustafa Ozsutcu ${ }^{\mathrm{c}}$
}

\begin{abstract}
The caroticocavernous fistula is a specific type of dural arteriovenous fistula characterized by abnormal arteriovenous shunting within the cavernous sinus. A caroticocavernous fistula results in high-pressure arterial blood entering the low-pressure venous cavernous sinus. This interferes with normal venous drainage patterns and compromises blood flow within the cavernous sinus and the orbit. We present a Barrow type A caroticocavernous fistula case, presented with glaucoma, ptosis and 6th nerve palsy without any history of trauma and atypically disappeared after acetolozomide theraphy. In conclusion, before beginning for an invasive treatment of a CCF, acetolozomide theraphy can be started initially because it may have a role in closure of CCF due to its vasoregulatory effects in the brain.
\end{abstract}

Keywords: CCF; Ptosis; Abducense nerve; Glaucoma

\section{Introduction}

Carotico-cavernous fistula (CCF) is an abnormal communication between carotid artery and cavernous sinus (CS). Fistulas are divided into four types: Type A are direct high-flow shunts between the internal carotid artery and the cavernous sinus; Type B are dural shunts between meningeal branches of the internal carotid artery and the cavernous sinus; Type $\mathrm{C}$ are dural shunts between meningeal branches of the external

\footnotetext{
Manuscript accepted for publication March 13, 2012

${ }^{a}$ Medipol University Ophthalmology Department, Istanbul, Turkey

${ }^{b}$ Private Ophthalmology Clinic, Istanbul, Turkey

'Ophthalmology Department, Bezmi Alem University Hospital, Turkey

${ }^{\mathrm{d}}$ Corresponding author: Goktug Demirci, Kocamustafapasa cad. Levent apt 128-2 34800 Cerrahpasa Fatih, Istanbul, Turkey.

Email: drgdemirci@yahoo.com

doi:10.4021/jmc601w
}

carotid artery and the cavernous sinus; and Type D are dural shunts between meningeal branches of both the internal and external carotid arteries and the cavernous sinus [1]. A CCF results in high-pressure arterial blood entering the low-pressure venous cavernous sinus. This interferes with normal venous drainage patterns and compromises blood flow within the cavernous sinus and the orbit. CCFs can be caused by trauma. They also can occur spontaneously. Most CCFs are of spontaneous origin and unknown etiology $[1,2]$.

\section{Case Report}

We report a CCF case with dilated episcleral vein, secondary open angle glaucoma, ptosis and 6th nerve palsy. A 65-yearold female presented with redness of left eye without any systemic illness and any history of trauma except previously known HT. Her ocular examination revealed dilated corkscrew episcleral veins, ptosis, proptosis, 6th nerve palsy, and a high intraocular pressure (IOP) of $45 \mathrm{mmHg}$ in the left eye. Right eye examination was completely normal (Fig. 1).

With these findings to rule out arteriovenous malformation, cavernous sinus thrombosis, cavernous sinus tumors, orbital tumors, skull base tumors and mucocele we performed cranial MRI and cranial CT angiography. MRI was normal. Barrow type A CCF was diagnosed in angiography.

We started antiglaucomatous treatment with systemic Acetolozomide (Diazomid®-Sanofi-Aventis) 3 x $250 \mathrm{mg}$, K tb (Kalinor ${ }^{\circledR}$ Abbott) daily, topical combined Dorzolomid Timolol Maleate (Cosopt ${ }^{\circledR}$ Merck Sharp \& Dohme) 2 times daily, topical Brimonidin Tartrate (Alphagan $P \circledR$ Allergan) 2 times daily and had appointment for closure of CCF from invasive neuroradiology department of the hospital for two weeks later.

In the literature there are two aproaches for glaucoma and CCF. First aproach is waiting for surgical closure of CCF and IOP decreases thereafter. The second is performing trabeculectomy without waiting [2]. While we were waiting for the closure of CCF with a very risky operation, at the end of two weeks IOP of the the eye dropped under $20 \mathrm{mmHg}$, abducens nerve pathology, proptosis and conjunctival chemosis disappeared. All the medications were withdrawn and 


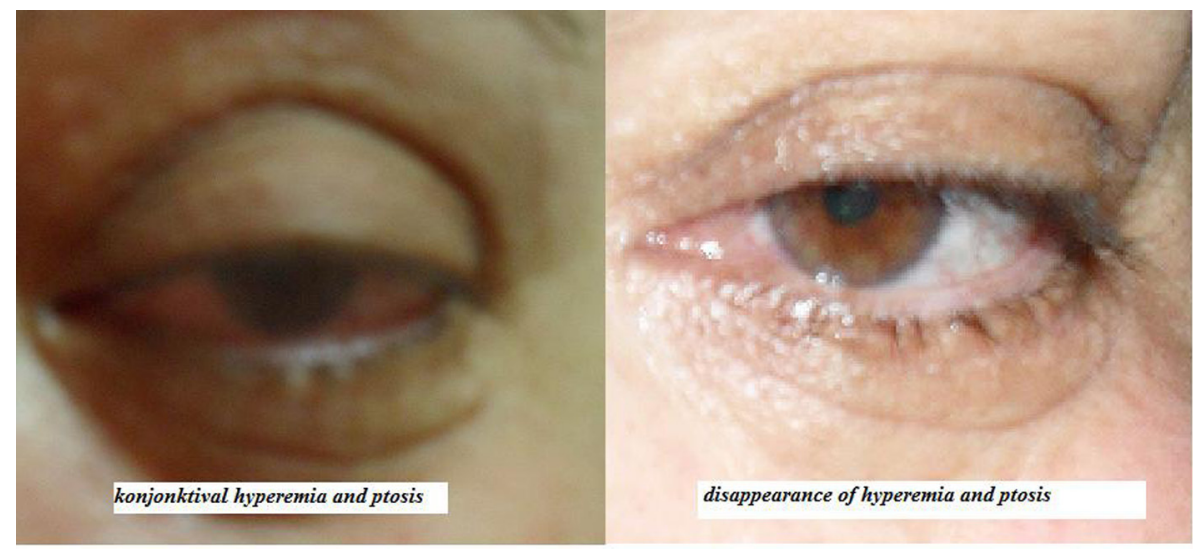

Figure 1. Left eye of the patient before and after disappearance of the CCF.

at last CCF closure documented angiographically. The most interesting finding was the patients response to acetolozomide as her feeling of decreased headache after taking the acetolozomide tablet.

\section{Discussion}

A caroticocavernous fistula results in high-pressure arterial blood entering the low-pressure venous cavernous sinus. This interferes with normal venous drainage patterns and compromises blood flow within the cavernous sinus and the orbit. Caroticocavernous fistulas represent approximately $12 \%$ of all dural arteriovenous fistulas. Type A is more common in young males. Types $\mathrm{B}, \mathrm{C}$, and $\mathrm{D}$ are more common in women older than 50 years, with a 7:1 female-to-male ratio $[3,4]$. The most common $(70 \%-90 \%)$ etiology of direct $\mathrm{CCF}$ is trauma from a basal skull fracture resulting in tear in the internal carotid artery (ICA) within the cavernous sinus. Causes that are not very common include spontaneous rupture of an existing aneurysm or atherosclerotic artery, usually in postmenopausal, hypertensive females [5].

Direct CCF has a frequency of up to $90 \%$ of all CCF and glaucoma is a potential complication of untreated direct CCF. This case was a Type A Direct CCF although spontaneous closure from thrombosis of CS is unlikely (especially those that occur after trauma or in high flow fistulas), CCF is closed spontaneously in our case [6]. There are many ways of treatment although most CCFs are not life threatening, but the involved eye is at risk. Main indications for treatment includes glaucoma, diplopia, intolerable bruit or headache, and severe proptosis causing exposure keratopathy $[5,6]$.

Surgical treatment has includes ligation of the external and internal carotid arteries; and fistula embolization with particles, glue, detachable balloons and thrombogenic microcoils [7]. Reported complications of the treatment include ophthalmic artery occlusion, cerebral infarction, increased proptosis, elevation of IOP, choroidal detachment that re- quired suprachoroidal drainage, venous stasis retinopathy, uncontrolled bleeding and central retinal vein occlusion [57].

Glaucoma is a potential complication which can appear in direct CCF. The most common form of glaucoma is caused by increased pressure in the episcleral veins [8]. Dilatation of the conjunctival vessels is the most obvious sign of this disease. Secondary glaucoma is a frequently observed ocular manifestation of CCF, and closure of the fistula is the primary condition required for favorable IOP control. Conjunctival chemosis appears in almost all patients diagnosed with direct carotidcavernous fistula, preceeding the exophthalmia in most cases. Diplopia is caused by damage of one or multiple nerves due to compression or ischemia; the most affected nerve is the abducens nerve as in our case, but also the trochlear nerve could be affected [9].

In our case beta adrenergic antagonists, topical and systemic carbonic anhydrase inhibitors and alpha 2 agonists have reduced the intraocular pressure. Question is what may be the reason of spontaneous closure of CCF in our case. Our opinion is the only reason may be the use of CAI inhibitor acetolozomide.

Sulphonamide CA inhibitors (CAIs) are useful as diuretics, or in the treatment and prevention of a variety of diseases such as glaucoma, epilepsy, congestive heart failure, mountain sickness, gastric and duodenal ulcers, neurological disorders and osteoporosis. Acetolozomide is a CAE inhibitor diuretic drug which effects on proximal tubules of kidney. Acetolozomide also has a local vasodilator effect on the cerebral arterioles, unrelated to its specific effects as a carbonic anhydrase inhibitor. Also acetalozomide has effect on cerebral vascular permeability [10]. Our opinion is these effects would lead to decrease in blood passage from ICA to sinus and spontaneus closure of the fistula.

We present this case because spontaneous closure of symptomatic direct CCF is uncommon. The case is also being presented to highlight acetolozomide theraphy that may be the reason of a spontaneous closure of a type A CCF, our 
opinion is acetolozomide as a vasoregulator drug acting on cerebral vasculature may have had a role in closure of $\mathrm{CCF}$ in our case.

\section{Conclusions}

Carotico-cavernous fistula (CCF) is a very dangerous neuroophthalmologic disorder. Diagnosis and treatment is really sophisticated. Acetolozomide as a CAI inhibitor may have a role in the treatment of this disorder.

\section{Grant Support}

There is no financial support from any of the organisation. The authors declare that there is not any financial or proprietary interest in any of the material.

\section{References}

1. Barrow DL, Spector RH, Braun IF, Landman JA, Tindall SC, Tindall GT. Classification and treatment of spontaneous carotid-cavernous sinus fistulas. J Neurosurg. 1985;62(2):248-256.

2. Chaudhry IA, Elkhamry SM, Al-Rashed W, Bosley TM. Carotid cavernous fistula: ophthalmological implications. Middle East Afr J Ophthalmol. 2009;16(2):57-63.

3. Murthy T.V.S.P, Mohan C, Gupta P. Carotococavernous
Fistula- A Case Report. Indian J. Anaesth. 2005; 49:22022.

4. Lewis AI, Tomsick TA, Tew JM, Jr. Management of 100 consecutive direct carotid-cavernous fistulas: results of treatment with detachable balloons. Neurosurgery. 1995;36(2):239-244; discussion 244-235.

5. Miller NR. Diagnosis and management of dural carotid-cavernous sinus fistulas. Neurosurg Focus. 2007;23(5):E13.

6. Goldberg RA, Goldey SH, Duckwiler G, Vinuela F. Management of cavernous sinus-dural fistulas. Indications and techniques for primary embolization via the superior ophthalmic vein. Arch Ophthalmol. 1996;114(6):707-714.

7. Preechawat $P$, Narmkerd P, Jiarakongmun P, Poonyathalang A, Pongpech SM. Dural carotid cavernous sinus fistula: ocular characteristics, endovascular management and clinical outcome. J Med Assoc Thai. 2008;91(6):852858 .

8. Talks SJ, Salmon JF, Elston JS, Bron AJ. Cavernous-dural fistula with secondary angle-closure glaucoma. Am J Ophthalmol. 1997;124(6):851-853.

9. Henderson JW, Schneider RC. The ocular findings in carotid-cavernous fistula in a series of 17 cases. Am J Ophthalmol. 1959;48:585-597.

10. Gao BB, Clermont A, Rook S, Fonda SJ, Srinivasan VJ, Wojtkowski M, Fujimoto JG, et al. Extracellular carbonic anhydrase mediates hemorrhagic retinal and cerebral vascular permeability through prekallikrein activation. Nat Med. 2007;13(2):181-188. 